\title{
Teaching diffraction and signal processing with a liquid crystal spatial light modulator
}

José Luis Martínez, Ignacio Moreno, Esmail Ahouzi

José Luis Martínez, Ignacio Moreno, Esmail Ahouzi, "Teaching diffraction and signal processing with a liquid crystal spatial light modulator," Proc. SPIE 9664, Ninth International Topical Meeting on Education and Training in Optics and Photonics, 966429 (24 October 2005); doi: 10.1117/12.2207775

SPIE Event: Ninth International Topical Meeting on Education and Training in Optics and Photonics, 2005, Marseille, France 


\title{
Ref ETOP089
}

\section{Teaching diffraction and signal processing with a liquid crystal spatial light modulator}

\author{
José Luis Martínez ${ }^{1}$, Ignacio Moreno ${ }^{1}$ and Esmail Ahouzi ${ }^{2}$
}

${ }^{1}$ Departamento de Ciencia y Tecnología de Materiales. Universidad Miguel Hernández de Elche, Alicante (Spain).

${ }^{2}$ Institut National des Postes et Telecomunications (INTP). Madinat Al Irfane, Rabat, (Morocco).

\begin{abstract}
In this work we propose the use of a liquid crystal spatial light modulator (LC-SLM) as a useful tool to teach and experience diffraction and signal processing. The LC-SLM acts as a programmable pixelated diffractive mask. The Fourier spectra of the image displayed in the LC-SLM is visualized through a simple free propagation diffraction experiment. This optical system allows easily testing different diffractive elements and performing several signal processing experiments. As a demonstration we include experimental results with diffraction gratings, computer generated holograms, diffractive lenses and axicons.
\end{abstract}

\section{Keywords \\ Diffraction, Signal Processing, Liquid-Crystal Displays}

\section{Introduction}

\section{Summary}

Diffraction is a classical subject taught extensively on Physics or Engineering degrees [1]. Within the Fraunhofer approximation, a Fourier transform relation links the diffraction pattern with the transmittance of the diffractive mask. Classical diffraction patterns that are studied in many textbooks are those produced by rectangular or circular apertures, or diffraction gratings [2]. However, the possibility that students test and even design their own diffraction masks is very interesting from the comprehensive point of view.

A liquid crystal spatial light modulator is an optoelectronic device commonly found in commercial projection displays. Under proper configuration of the external polarization elements, these devices can produce an amplitude-only or a phase-only modulation versus the addressed gray level [3]. Figure 1 shows the experimental setup, where we used a LCSLM from CRL-Optics placed between a polarizer-waveplate pair. The proper orientation of these polarization elements makes the display act as a phase-only or amplitude-only mask.

This possibility makes this device very interesting in applications in diffractive optics. The diffractive mask can be programmed in real time from a computer. Therefore, once the optical system has been aligned, the students can easily produce different diffraction patterns simply by modifying the image that is being addressed to the display. Very different diffractive elements can

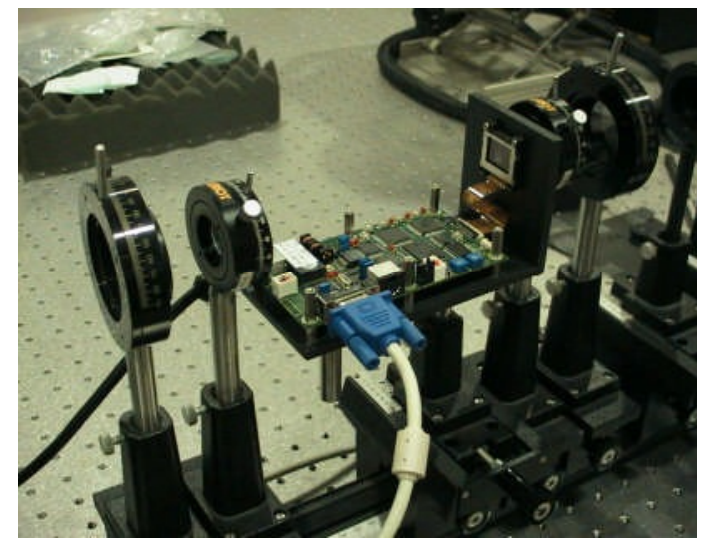

Figure 1. Experimental setup. 
be designed and experienced with the same optical setup.

\section{Experimental diffraction results}

In figure 2 we present some of the possibilities. Figure 2(a) corresponds to the case when a uniform gray level image is addressed to the LC-SLM screen. Since the device is pixelated, it acts as two-dimensional grating. The diffraction pattern consists in the typical square grid of the diffracted orders. Figure 2(b) corresponds to the case when a computer generated hologram is displayed. The computer generated hologram is a kinoform i.e., the phase of the Fourier transform of the input object (in this case a butterfly). The reconstruction shows the typical edge enhanced version of the input object [4]. Let us note that the Fourier transform spectrum obtained optically consists on different replicas of the kinoform reconstruction. They are centred at the locations of the diffraction orders shown in Fig. 1(a). These replicas are the convolution of the original reconstruction of the kinoform and the diffraction orders generated by the pixelated structure of the display. This is a very nice visualization of the two dimensional Whittaker-Shannon sampling theorem [2] which evidences the limited spatial sampling produced by the pixelization of the device.

In figure 2(c) a binary amplitude grating is addressed to the LC-SLM. The grating is oriented horizontally, so the typical diffracted orders appear vertically. Again, the set of diffracted orders can be viewed as the convolution of those generated by the pixelated structure with those generated by the addressed grating. Finally, Fig. 2(d) corresponds to the case when an axicon [5] is displayed on the LCD. The reconstruction consists in concentric circles around every diffraction order. For this diffractive element the sampling limit imposed by the pixelization is not enough, and the reconstructions overlap.

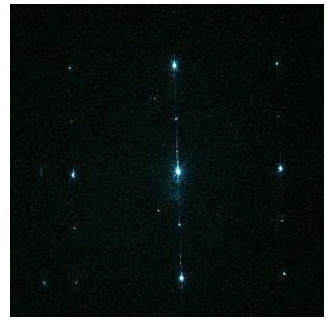

(a)

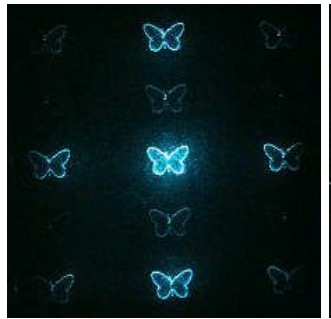

(c) (d)

d)

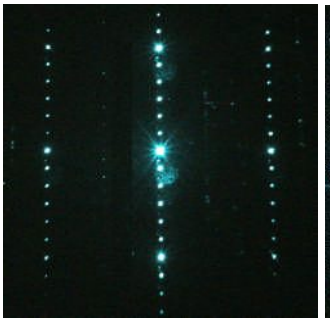

Figure 2. Experimental diffraction patterns generated by: (a) A uniform image, (b) a phase only computer generated hologram, (c) a grating and (d) a binary axicon.

The real-time flexibility that the LC-SLM provides permits to design and test amazing optical diffractive masks. As an example in figure 3 we show a diffractive phase-only mask which is obtained by combining a phase-only computer generated hologram with a Fresnel lens. We add the two phase functions and we display the resulting function on the LCD [6].

In the four cases shown in Fig. 3 we add Fresnel lenses with different focal lengths to the same phase-only computer generated hologram. In this figure we only show the central reconstruction. Because of the different quadratic phase factors of each Fresnel lens, the reconstruction appears in different planes and with different size. In addition, another interesting effect happens due to the resolution limit of the display. If the quadratic phase varies fast enough, the inherent sampling due to the pixelated structure causes a multi-focus effect on the Fresnel lens [7]. Therefore the reconstruction consists on different replicas of the original reconstruction. The shorter the focal length of the Fresnel lens, the larger the number of replicas generated. This shows another amazing consequence of the sampling theorem, in this case applied to the quadratic phase of a Fresnel lens. 


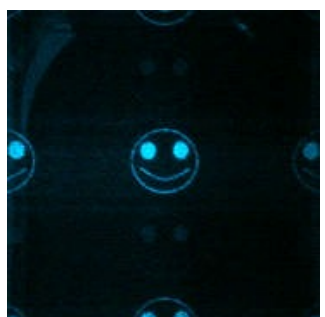

(a)

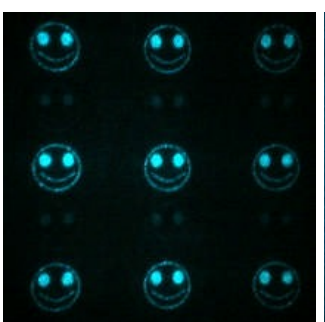

(d)
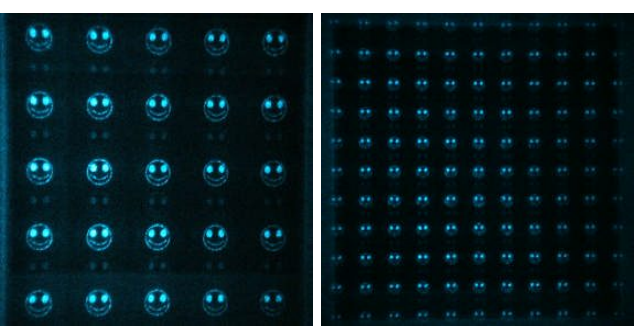

Figure 3. Experimental diffraction patterns generated by a phase-only diffractive mask obtained by adding the phase of a computer generated hologram with the quadratic phase characteristic of a Fresnel lens. From left to right the focal length of the Fresnel lens is decreased.

\section{Conclusion}

In summary, the LC-SLM is a very interesting optical device useful to experience many diffraction and signal processing effects. Therefore it can be a very useful tool to teach and experience these subjects.

\section{Acknowledgements}

We acknowledge finantial support from Ministerio de Ciencia y Tecnología from Spain (ref: BFM2003-06273-C02-02/FISI) and from Agencia Española de Cooperación Internacional $\mathrm{AECI}$ (ref.: 82/03/P).

\section{References}

[1] E. Hecht, Optics, 4th edition, Addison-Wesley, Reading MA (1997).

[2] J. Goodman, Introduction to Fourier Optics, McGraw-Hill (1996).

[3] A. Márquez, C. lemmi, I. Moreno, J. A. Davis, J. Campos and M. J. Yzuel, "Quantitative prediction of the modulation behavior of twisted nematic liquid crystal displays," Optical Engineering 40, 2558-2564 (2001).

[4] I. Moreno, J. Campos, C. Gorecki and M. J. Yzuel, "Effects of amplitude and phase mismatching errors in the generation of a kinoform for pattern recognition," Japanese Journal of Applied Physics 34, 6423-6434 (1995).

[5] Z. Jaroszewicz, A. Burvall and A. Friberg, "Axicon - the most important optical element", Optics \& Photonics News pp. 34-39, April (2005).

[6] J. A. Davis, D. M. Cottrell, R. A. Lilly and S. W. Connely, "Multiplexed phase-encoded lenses written on spatial light modulators," Optics Letters 14, 420-422 (1989)

[7] E. Carcolé, J. Campos and S. Bosch, "Diffraction theory of Fresnel lenses encoded in low-resolution devices", Applied Optics 33, 162-174 (1994) 